\title{
On Proof Nets for Multiplicative Linear Logic with Units
}

\author{
Lutz Straßburger and François Lamarche \\ INRIA-Lorraine, Projet Calligramme \\ 615, rue du Jardin Botanique - 54602 Villers-lès-Nancy — France \\ \{Lutz.Strassburger, Francois.Lamarche\}@loria.fr
}

\begin{abstract}
In this paper we present a theory of proof nets for full multiplicative linear logic, including the two units. It naturally extends the well-known theory of unit-free multiplicative proof nets. A linking is no longer a set of axiom links but a tree in which the axiom links are subtrees. These trees will be identified according to an equivalence relation based on a simple form of graph rewriting. We show the standard results of sequentialization and strong normalization of cut elimination. Furthermore, the identifications enforced on proofs are such that the proof nets, as they are presented here, form the arrows of the free (symmetric) *-autonomous category.
\end{abstract}

\section{Introduction}

For a long time formal logicians have been aware of the need to determine, given a formal system $\mathcal{S}$ and two proofs of a formula $A$ in that system, when these two proofs are "the same" proof. As a matter of fact this was already a concern of Hilbert when he was preparing his famous lecture in 1900 Thi03. This problem has taken more importance during the last few years, because many logical systems permit a close correspondence between proofs and programs.

In a formalism like the sequent calculus (and to a lesser degree, natural deduction), it is oftentimes very easy to see that two derivations $\pi_{1}$ and $\pi_{2}$ should be identified because $\pi_{1}$ can be transformed in to $\pi_{2}$ by a sequence of rule permutations that are obviously trivial. It is less immediately clear in general what transformations can be effected on a proof without changing its essence. But here category theory is very helpful, providing criteria for the identification of proofs that are simple, general and unambiguous, if sometimes too strong Gir91.

The advent of linear logic marked a significant advance in that quest. In particular the multiplicative fragment of linear logic comes equipped with an extremely successful theory of proof identification: not only do we know exactly when two sequent proofs should be identified (the allowed rule permutations are described in [Laf95]), but there is a class of simple formal objects that precisely represent these equivalence classes of sequent proofs. These objects are called proof nets, and they have a strong geometric character, corresponding to additional graph structure ("axiom links") on the syntactical forest of the sequent. More precisely, given a sequent $\Gamma=A_{1}, \ldots, A_{n}$ and a proof $\pi$ of that sequent, 
then the proof net that represents $\pi$ is simply given by the syntactical forest of $\Gamma$ decorated with additional edges (shown in thick lines) that represent the identity axioms that appeared in the proof:

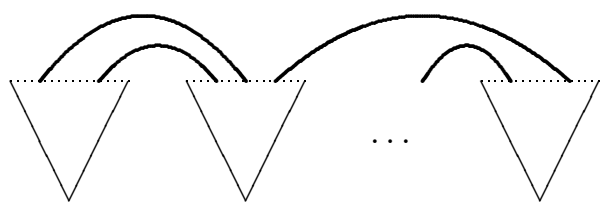

Moreover proof nets are vindicated by category theory, since the category of two-formula sequents and proof nets is precisely the free $*$-autonomous category [Bar79] (without units) on the set of generating atomic formulas Blu93]. As a matter of fact axiom links were already visible, under the name of Kelly-Mac Lane graphs in the early work KL71 that tried to describe free autonomous categories; Girard's key insights Gir87] here were in noticing that there was an inherent symmetry that could be formalized through a negation (thus the move from autonomous to $*$-autonomous), and that the addition of the axiom links to the sequent's syntactic forest were enough to completely characterize the proof.

The theory of proof nets has been extended to larger fragments of linear logic; when judged from the point of view of their ability to identify proofs that should be identified, these extensions can be shown to have varying degrees of success. One of these extensions, which complies particularly well with the categorical ideal, is the inclusion of additive connectives presented in [HvG03, in which the additives correspond exactly to categorical product and coproduct.

In this paper we give a theory of proof nets for the full multiplicative fragment. That is, our theory of proof nets includes the multiplicative units. We prove that it allows us to construct the free $*$-autonomous category with units on a given set of generating objects, thus getting full validation from the categorical imperative.

There are only two other presentations for multiplicative units that we are aware of. In KO99, the authors provide an internal language for autonomous

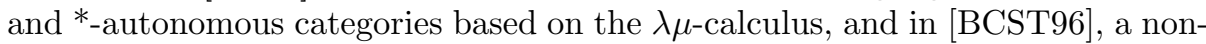
standard version of two-sided proof nets for a weaker logic is developed from which the authors also claim to have constructed free $*$-autonomous categories. Our approach is different in the following way: By making full use of the symmetry given by the combination of an involutive negation and a one-sided sequent calculus, we get a notion of proof net which is considerably simpler than the one provided in BCST96.

\section{The Main Problem}

We assume that the reader is familiar with the sequent calculus for classical multiplicative linear logic.

The theory of $*$-autonomous categories tells us that whenever a proof contains a rule instance $r$ which appears after a $\perp$-introduction rule and which does 
(1)

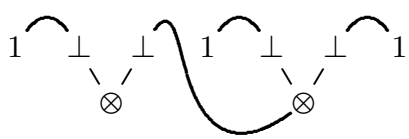

(2)

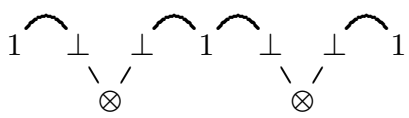

(3)

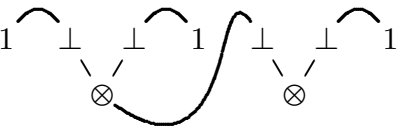

(4)

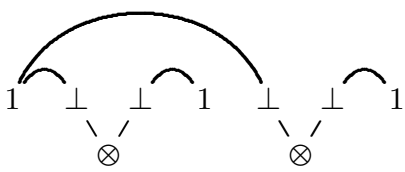

(5)

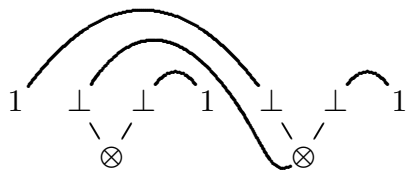

(6)

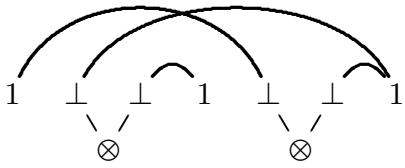

Fig. 1. Different representations of the same proof

not introduce a connective under that $\perp$, then $r$ can be pushed above that $\perp$ introduction without changing the proof:

$$
\stackrel{\perp \frac{\Gamma}{\perp, \Gamma} \cdots}{\perp, \Gamma^{\prime}} \quad \longleftrightarrow \quad \mathrm{r} \frac{\Gamma \quad \cdots}{\perp \frac{\Gamma^{\prime}}{\perp, \Gamma^{\prime}}}
$$

This seemingly trivial permutation actually has deep consequences. Supposing that rule $r$ was a $\otimes$-introduction, there is now a choice of two branches on which to do the $\perp$-introduction.

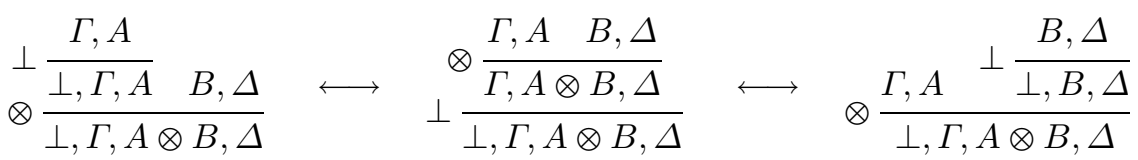

Ordinary proof nets for multiplicative linear logic are characterized by the presence of links, which connect the atoms of the syntactical forest of the sequent. When extending them to multiplicative units, the first impulse is probably to try to attach the $\perp$ s that are present on the sequent forest on other atomic formulas. This is what is done in BCST96] and corresponds, in the sequent calculus, to doing the $\perp$-introductions as early as possible, that is, as high up on the sequent tree as can be done. The paragraph above shows that an arbitrary choice has to be made because of tensor introductions: in a $\otimes$-intro one branch of the sequent proof tree or the other has to be chosen for doing the $\perp$-intro. In such a situation correct identification of proofs can only be achieved by considering equivalence classes of graphs, and the theory of proof nets involves an equivalence relation on a set of "correct" graphs. 
Another possibility is to attach these $\perp_{\mathrm{s}}$ "as low as possible" on the forest, corresponding to the idea that in the sequent calculus deduction the $\perp$-intro would be done as late as possible, for example just before the $\perp$ instance gets a connective introduced under it. One way of implementing this is linking the $\perp$ instance to the last connective that was introduced above it. This is not the only way of doing things, for example we could imagine links that attach that $\perp$ instance to several subformulas of the sequent forest, corresponding to the several conclusions of the sequent that existed above the $\perp$-introduction.

But whatever way we choose to "normalize" proofs, we claim that if the conventional notion of "link" is used for $\perp$ s (i.e., if we consider a proof $\pi$ on the sequent $\Gamma$ as the sequent forest of $\Gamma$ decorated with special edges that encode information about the essence of $\pi$ ) we still need to use equivalence classes of such graphs, and there is no hope of having a normal form in that universe of enriched sequent graphs. For instance, the six graphs in Figure 1 are easily seen to represent equivalent proofs, because going from an odd-numbered example to its successor is just sliding a $\perp$-intro up in one of the $\otimes$-intro branches, and going from an even-numbered example to its successor is just doing the reverse transformation. But notice that examples (3) and (5) are distinct but isomorphic graphs, since one can be exactly superposed on the other by only using the Exchange rule. Thus it is impossible, given the information at our disposal, to choose one instead of the other to represent the abstract proof they both denote. The only way this could be done would be by using arbitrary extra information, like the order of the formulas in the sequent, a strategy that only replaces the overdeterminism of the sequent calculus by another kind of overdeterminism.

The same can be said of Examples (2) and (6), which are also isomorphic modulo Exchange. But notice that these two comply to the "as early as possible" strategy, while the previous two were of the "as late as possible" kind. So for neither strategy can there be a hope a graphical normal form. The interested reader can verify that the six examples above are part of a "ring" of 24 graphs that are all equivalent from the point of view of category theory.

Thus there is one aspect of our work that does not differ from [BCST96, which is our presentation of abstract proofs as equivalence classes of graphs. But some related aspects are significantly different:

- The graphs that belong to our equivalence classes are standard multiplicative proof nets, where the usual notions, like correctness criteria and the empire of a tensor branch, will apply. It is just that some $\varnothing$ and $\otimes$ links are used in a particular fashion to deal with the units. (The readers can choose their favorite correctness exiterion since they are all equivalent; in this paper we will use the one of DR89 because of its popularity.)

- The equivalence relation we will present is based on a very simple set of rewriting rules on proof graphs. As a matter of fact, there is only one nontrivial rule, since the other rules have to do with commutativity and associativity of the connectives and can be dispensed with if we use, for example, $n$-ary connectives. 

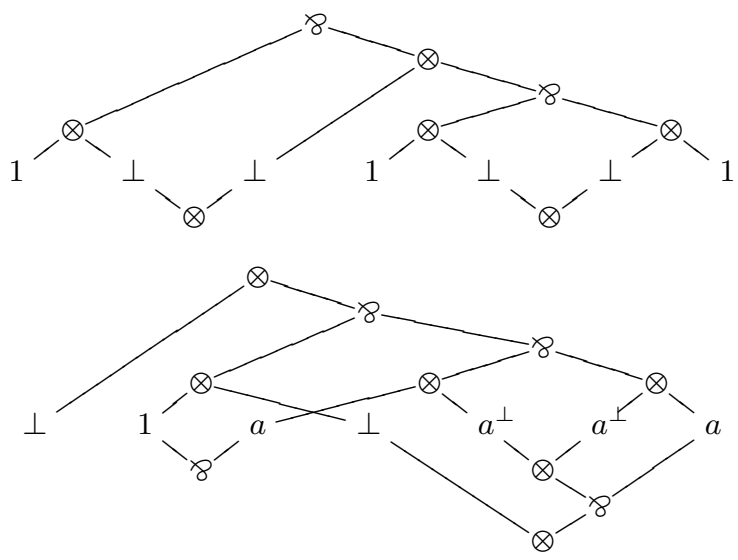

Fig. 2. Two examples of proof graphs

\section{Cut Free Proof Nets for MLL}

Let $\mathcal{A}=\{a, b, \ldots\}$ be an arbitrary set of atoms, and let $\mathcal{A}^{\perp}=\left\{a^{\perp}, b^{\perp}, \ldots\right\}$. The set of MLL formulas is defined as follows:

$$
\mathcal{F}::=\mathcal{A}\left|\mathcal{A}^{\perp}\right| 1|\perp| \mathcal{F} \otimes \mathcal{F} \mid \mathcal{F} \otimes \mathcal{F} .
$$

Additionally, we will define the set of MLL linkings (which can be seen as a special kind of formulas) as follows:

$$
\mathcal{L}::=1\left|a \otimes a^{\perp}\right| a^{\perp} \otimes a|\perp \otimes \mathcal{L}| \mathcal{L} \otimes \perp \mid \mathcal{L} \otimes \mathcal{L}
$$

Here, $a$ stands for any element of $\mathcal{A}$. We will use $A, B, \ldots$ to denote formulas, and $P, Q, \ldots$ to denote linkings. Sequents (denoted by $\Gamma, \Delta, \ldots$ ) are finite lists of formulas (separated by comma).

In the following, we will always consider both formulas and linkings as binary trees (and sequents as forests), whose leaves are decorated by elements of $\mathcal{A} \cup$ $\mathcal{A}^{\perp} \cup\{1, \perp\}$, and whose inner nodes are decorated by 8 or $\otimes$. We can also think of the nodes being decorated by the whole subformula above that node.

2.1 Definition. A pre-proof graph is a graph consisting of a linking $P$ and a sequent $\Gamma$, both of which share the same set of leaves. It will be denoted as $P \triangleright \Gamma$.

Following the tradition, we will draw these graphs such that the roots of the formula trees are at the bottom, the root of the linking tree is at the top, and the leaves are in between. Figure 2 shows two examples. The first of them corresponds to the first graph in Figure 1. A more compact notation for this is

$$
\begin{gathered}
\left(1_{1} \otimes \perp_{2}\right) \ngtr\left(\perp_{3} \otimes\left(\left(1_{4} \otimes \perp_{5}\right) \ngtr\left(\perp_{6} \otimes 1_{7}\right)\right)\right) \\
\nabla \\
1_{1}, \perp_{2} \otimes \perp_{3}, 1_{4}, \perp_{5} \otimes \perp_{6}, 1_{7}
\end{gathered}
$$


and

$$
\begin{gathered}
\perp_{1} \otimes\left(\left(1_{2} \otimes \perp_{4}\right) 8\left(\left(a_{3} \otimes a_{5}^{\perp}\right) \ngtr\left(a_{6}^{\perp} \otimes a_{7}\right)\right)\right) \\
\quad \nabla \\
\perp_{1}, 1_{2} 8 a_{3}, \perp_{4} \otimes\left(\left(a_{5}^{\perp} \otimes a_{6}^{\perp}\right) \ngtr a_{7}\right)
\end{gathered}
$$

Here, the indices are used to show how the leaves of the linking and the leaves of the sequent are identified. In this way we will, throughout this paper, use indices on atoms to distinguish between different occurrences of the same atom (i.e. $a_{3}$ and $a_{7}$ do not denote different atoms). In the same way, indices on the units 1 and $\perp$ are used to distinguish different occurrences.

2.2 Definition. A switching of a pre-proof graph $P \triangleright \Gamma$ is a graph $G$ that is obtained from $P \triangleright \Gamma$ by omitting for each 8 -node one of the two edges that connect the node to its children. DR89]

2.3 Definition. A pre-proof graph $P \triangleright \Gamma$ is called correct if all its switchings are connected and acyclic. A proof graph is a correct pre-proof graph.

The examples in Figure 2 are proof graphs.

Let $P \triangleright \Gamma$ be a pre-proof graph where one $\perp$ is selected. Let it be indexed as $\perp_{i}$. Now, let $G$ be a switching of $P \triangleright \Gamma$, and let $G^{\prime}$ be the graph obtained from $G$ by removing the edge between $\perp_{i}$ and its parent in $P$ (which is always $\mathrm{a} \otimes)$. Then $G^{\prime}$ is called an extended switching of $P \triangleright \Gamma$ with respect to $\perp_{i}$. Observe that, if $P \triangleright \Gamma$ is correct, then every extended switching is a graph that has exactly two connected components.

We will use the notation $P\{Q\} \triangleright \Gamma$ to distinguish the subtree $Q$ of the linking tree of the graph. Then $P\{\}$ is the context of $Q$.

2.4 Equivalence on Pre-proof Graphs. On the set of pre-proof graphs we will define the relation $\sim$ to be the smallest equivalence relation satisfying

$$
\begin{aligned}
& P\{Q \ngtr R\} \triangleright \Gamma \sim P\{R \ngtr Q\} \triangleright \Gamma \\
& P\{(Q \ngtr R) \ngtr S\} \triangleright \Gamma \sim P\{Q \ngtr(R \ngtr S)\} \triangleright \Gamma \\
& P\{Q \otimes R\} \triangleright \Gamma \sim P\{R \otimes Q\} \triangleright \Gamma \\
& \left.P\left\{\perp_{i} \otimes\left(Q \otimes \perp_{j}\right)\right\} \triangleright \Gamma \sim P\left\{\left(\perp_{i} \otimes Q\right) \otimes \perp_{j}\right)\right\} \triangleright \Gamma \\
& \left.P\left\{Q \ngtr\left(R \otimes \perp_{i}\right)\right\} \triangleright \Gamma \stackrel{(*)}{\sim} P\left\{(Q \ngtr R) \otimes \perp_{i}\right)\right\} \triangleright \Gamma \quad,
\end{aligned}
$$

where the last equation only holds if the following side condition is fulfilled:

(*) In each extended switching of $P\left\{Q \ngtr\left(R \otimes \perp_{i}\right)\right\} \triangleright \Gamma$ with respect to $\perp_{i}$ no node of the subtree $Q$ is connected to $\perp_{i}$.

The following proof graph is equivalent to the second one in Figure 2:

$$
\begin{gathered}
\left(\left(\left(\perp_{1} \otimes 1_{2}\right) \otimes \perp_{4}\right) 8\left(a_{3} \otimes a_{5}^{\perp}\right)\right) 8\left(a_{6}^{\perp} \otimes a_{7}\right) \\
\nabla \\
\perp_{1}, 1_{2} 8 a_{3}, \perp_{4} \otimes\left(\left(a_{5}^{\perp} \otimes a_{6}^{\perp}\right) \ngtr a_{7}\right) .
\end{gathered}
$$




$$
\begin{array}{cc}
\text { id } \frac{\operatorname{ex} \frac{P \triangleright \Gamma, A, B, \Delta}{P \triangleright \Gamma, B, A, \Delta}}{a \otimes a^{\perp} \triangleright a, a^{\perp}} & \perp \frac{P \triangleright \Gamma}{\perp \otimes P \triangleright \perp, \Gamma} \\
1 \frac{P \triangleright 1}{1 \triangleright A, B, \Gamma} & \quad \otimes \frac{P \triangleright \Gamma, A \quad Q \triangleright B, \Delta}{P \ngtr Q \triangleright \Gamma, A \otimes B, \Delta}
\end{array}
$$

Fig. 3. Translation of cut free sequent calculus proofs into pre-proof graphs

2.5 Definition. A pre-proof net 1 is an equivalence class $[P \triangleright \Gamma]_{\sim}$. A preproof net is correct if one of its elements is correct. In this case it is called a proof net.

In the following, we will for a given proof graph $P \triangleright \Gamma$ write $[P \triangleright \Gamma]$ to denote the proof net formed by its equivalence class (i.e. we will omit the $\sim$ subscript).

2.6 Lemma. If $P \triangleright \Gamma$ is correct and $P \triangleright \Gamma \sim P^{\prime} \triangleright \Gamma$, then $P^{\prime} \triangleright \Gamma$ is also correct.

Proof: That the first four equations preserve correctness is obvious. If in the last equation there is a switching that makes one side disconnected, then it also makes the other side disconnected. For acyclicity, we have to check whether there is a switching that produces a cycle on the right-hand side of the equation and not on the left-hand side. This is only possible if the cycle contains some nodes of $Q$ and the $\perp_{i}$. But this case is ruled out by the side condition (*).

Lemma 2.6 ensures that the notion of proof net is well-defined, in the sense that all its members are proof graphs, i.e. correct.

\section{$3 \quad$ Sequentialization}

Figure 3 shows how cut free sequent proofs of MLL can be inductively translated into pre-proof graphs.

We will call a pre-proof net sequentializable if one of its representatives can be obtained from a sequent calculus proof via this translation.

3.1 Theorem. A pre-proof net is sequentializable iff it is a proof net.

For the proof we will need the observation that any proof graph is an ordinary unit-free proof net, and the well-known fact that there is always a splitting tensor in such a net.

3.2 Observation Every proof graph $P \triangleright \Gamma$ is an ordinary unit-free proof net in the style of [DR89]. To make this precise, define for the linking $P$ the linking formula $P^{\star}$ inductively as follows:

\footnotetext{
${ }^{1}$ What we call pre-proof net is in the literature often called proof structure.
} 


$$
\begin{aligned}
a^{\perp \star}=a & 1^{\star}=\perp & & (A \otimes B)^{\star}=A^{\star} \otimes B^{\star} \\
a^{\star}=a^{\perp} & \perp^{\star}=1 & & (A \& B)^{\star}=A^{\star} 8 B^{\star}
\end{aligned}
$$

In other words, $P^{\star}$ is obtained from $P$ by replacing each leaf by its dual and by leaving all inner nodes unchanged. We now connect the leaves of $P^{\star}$ and $\Gamma$ by ordinary axiom links according to the leaf identification in $P \triangleright \Gamma$. If we forget the fact that $\perp$ and 1 are the units and think of them as ordinary dual atoms, then we have an ordinary unit-free proof net ${ }^{2}$.

3.3 Lemma. If in a unit-free proof net all roots are $\otimes$-nodes, then one of them is splitting, i.e. by removing it the net becomes disconnected. Gir87.

Proof of Theorem 3.1 (Sketch): It is easy to see that the rules 1 and id give proof graphs and that the rules $\perp, \varnothing$, and $\otimes$ preserve the correctness. Therefore every sequentializable pre-proof net is correct.

For the other direction pick one representative $P \triangleright \Gamma$ of the proof net and proceed by induction on the sum of the number of $\otimes$-nodes in the graph and the number of 8 -nodes in $\Gamma$. We now interpret $P \triangleright \Gamma$ as an ordinary unit-free proof net (according to Observation 3.2), and remove all 8 -roots (for those inside $\Gamma$ apply the 8 rule and proceed by induction hypothesis). Then apply Lemma 3.3. If the splitting $\otimes$ is inside $\Gamma$, we can apply the $\otimes$-rule and proceed by induction hypothesis; if it is inside $P$, it must come from an axiom link or a bottom link. In both cases we can obtain two smaller proof graphs, to which we can apply the induction hypothesis to get two sequent proofs, which can be composed by plugging one into a leaf of the other.

\section{Cut and Cut Elimination}

A cut is a formula $A \oplus A^{\perp}$, where $(1)$ is called the cut connective, and where the function $(-)^{\perp}$ is defined on formulas as follows (with a little abuse of notation):

$$
\begin{aligned}
& a^{\perp \perp}=a \quad 1^{\perp}=\perp \quad(A \otimes B)^{\perp}=A^{\perp} 8 B^{\perp} \\
& a^{\perp}=a^{\perp} \quad \perp^{\perp}=1 \quad(A \ngtr B)^{\perp}=A^{\perp} \otimes B^{\perp}
\end{aligned}
$$

A sequent with cuts is a sequent where some of the formulas are cuts. But cuts are not allowed to occur inside formulas, i.e. all $(1$-nodes are roots. A preproof graph with cuts is a pre-proof graph $P \triangleright \Gamma$, where $\Gamma$ may contain cuts. The (1)-nodes have the same geometric behavior as the $\otimes$-nodes. Therefore the correctness criterion stays literally the same, and we can define proof graphs with cuts and proof nets with cuts accordingly. In the translation from sequent proofs containing the cut rule into pre-proof graphs with cuts, the cut is treated as follows:

$$
\text { cut } \frac{\Gamma, A \quad A^{\perp}, \Delta}{\Gamma, \Delta} \leadsto \operatorname{cut} \frac{P \triangleright \Gamma, A \quad Q \triangleright A^{\perp}, \Delta}{P \ngtr Q \triangleright \Gamma, A \oplus A^{\perp}, \Delta} \text {. }
$$

${ }^{2}$ If $\Gamma$ consists of only one formula, then we have an object which is in BC99 called a bipartite proof net. In fact, two proof graphs (in our sense) are equivalent if and only if the two linkings (seen as formulas) are isomorphic (in the sense of [BC99]). 

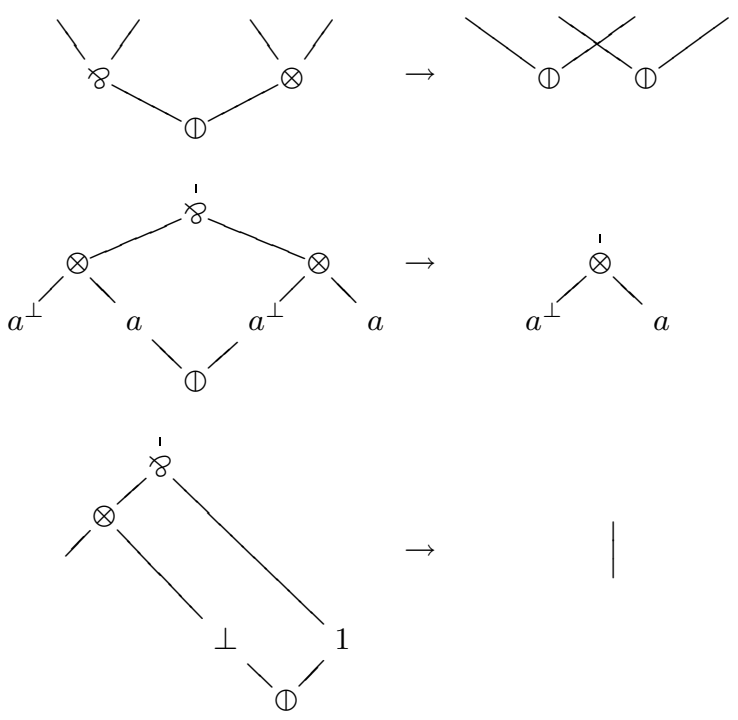

Fig. 4. Cut elimination reduction steps

Since the $(1)$ behaves in the same way as the $\otimes$, we immediately have the generalization of the sequentialization:

4.1 Theorem. A pre-proof net with cuts is sequentializable if and only if it is correct, i.e. it is a proof net with cuts.

On the set of cut pre-proof graphs we can define the cut reduction relation $\rightarrow$ as follows:

$$
\begin{array}{ccc}
P & & P \\
\nabla & \rightarrow & \nabla \\
(A \ngtr B) \Phi\left(A^{\perp} \otimes B^{\perp}\right), \Gamma & & A \oplus A^{\perp}, B \Phi B^{\perp}, \Gamma \\
P\left\{\left(a_{h}^{\perp} \otimes a_{i}\right) 8\left(a_{j}^{\perp} \otimes a_{k}\right)\right\} & & P\left\{a_{h}^{\perp} \otimes a_{k}\right\} \\
\nabla & \rightarrow & \nabla \\
a_{i} \Phi a_{j}^{\perp}, \Gamma & & \Gamma \\
P\left\{\left(Q \otimes \perp_{i}\right) \otimes 1_{j}\right\} & & P\{Q\} \\
\nabla & \rightarrow & \nabla \\
\perp_{i} \Phi 1_{j}, \Gamma & & \Gamma
\end{array}
$$

These reduction steps are shown in graphical notation in Figure 4 .

4.2 Lemma. If $P \triangleright \Gamma$ is correct and $P \triangleright \Gamma \rightarrow P^{\prime} \triangleright \Gamma^{\prime}$, then $P^{\prime} \triangleright \Gamma^{\prime}$ is also correct.

Proof: It is impossible that a cut reduction step introduces a cycle in a switching or makes it disconnected. 
Observe that it can happen that in a proof graph no reduction is possible, although there are cuts present in the sequent. For example, in

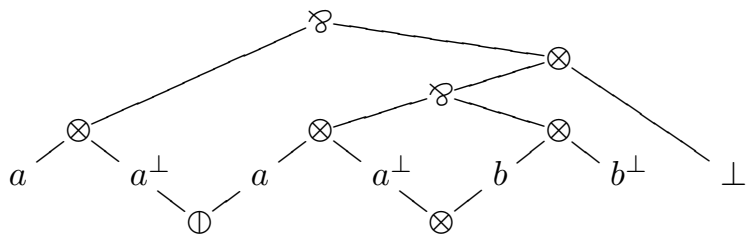

the cut cannot be reduced.

In a given proof graph $P \triangleright \Gamma$, a $(1$-node that can be reduced will be called ready. Obviously, a cut on a $\otimes$ - 8 -pair is always ready, but for a cut on atoms or units this is not necessarily the case, as the example above shows. However, we have the following theorem:

4.3 Theorem. Given a proof graph $P \triangleright \Gamma$ and a $(1-$-node in $\Gamma$, there is an equivalent proof graph $P^{\prime} \triangleright \Gamma$, in which that $(\mathbb{D}$-node is ready, i.e. can be reduced.

This is an immediate consequence of the following two lemmas.

4.4 Lemma. For every proof graph $P \triangleright a_{i} \oplus a_{j}^{\perp}, \Gamma$ that contains an atomic cut, there is an equivalent proof graph $P^{\prime}\left\{\left(a_{h}^{\perp} \otimes a_{i}\right) \otimes\left(a_{j}^{\perp} \otimes a_{k}\right)\right\} \triangleright a_{i} \oplus a_{j}^{\perp}, \Gamma$.

4.5 Lemma. For every proof graph $P \triangleright \perp_{i}\left(11_{j}, \Gamma\right.$ that contains a cut on the units, there is an equivalent proof graph $P^{\prime}\left\{\left(Q \otimes \perp_{i}\right) \diamond 1_{j}\right\} \triangleright \perp_{i}\left(\mathbb{1} 1_{j}, \Gamma\right.$.

For proving them, we will use the following three lemmas.

4.6 Lemma. Let $P\left\{\left(\perp_{k} \otimes R\left\{x_{i}\right\}\right) \diamond\left(S\left\{x_{j}^{\perp}\right\} \otimes \perp_{h}\right)\right\} \triangleright x_{i} \oplus x_{j}^{\perp}, \Gamma$ be a proof graph, where $x$ is an arbitrary atom or a unit, and $x^{\perp}$ its dual.

Then at least one of $P\left\{\perp_{k} \otimes\left(R\left\{x_{i}\right\} \otimes\left(S\left\{x_{j}^{\perp}\right\} \otimes \perp_{h}\right)\right)\right\} \triangleright x_{i} \oplus x_{j}^{\perp}, \Gamma$ and $P\left\{\left(\left(\perp_{k} \otimes R\left\{x_{i}\right\}\right) \& S\left\{x_{j}^{\perp}\right\}\right) \otimes \perp_{h}\right\} \triangleright x_{i} \oplus x_{j}^{\perp}, \Gamma$ is equivalent to it.

4.7 Lemma. Let $P\left\{\left(\perp_{k} \otimes R\left\{x_{i}\right\}\right) \diamond\left(x_{j}^{\perp} \otimes Q\right)\right\} \triangleright x_{i} \oplus x_{j}^{\perp}, \Gamma$ be a proof graph, where $x$ is an arbitrary atom or a unit, and $x^{\perp}$ its dual. Then $P\left\{\perp_{k} \otimes\left(R\left\{x_{i}\right\} \ngtr\left(x_{j}^{\perp} \otimes Q\right)\right)\right\} \triangleright x_{i} \oplus x_{j}^{\perp}, \Gamma$ is equivalent to it.

4.8 Lemma. Let $P\left\{\left(\perp_{k} \otimes R\left\{x_{i}\right\}\right) \ngtr x_{j}^{\perp}\right\} \triangleright x_{i} \oplus x_{j}^{\perp}, \Gamma$ be a proof graph, where $x$ is an arbitrary atom or a unit, and $x^{\perp}$ its dual.

Then $P\left\{\perp_{k} \otimes\left(R\left\{x_{i}\right\} \diamond x_{j}^{\perp}\right)\right\} \triangleright x_{i} \oplus x_{j}^{\perp}, \Gamma$ is equivalent to it.

Proof of Lemma 4.4 (Sketch): Since the proof graph is correct, the linking $P$ must be of the shape $P^{\prime \prime}\left\{R\left\{a_{h}^{\perp} \otimes a_{i}\right\} \& S\left\{a_{j}^{\perp} \otimes a_{k}\right\}\right\}$ for some contexts $P^{\prime \prime}\{\}$, $R\{\}$ and $S\{\}$. The contexts $R\{\}$ and $S\{\}$ can be reduced to \{\} by applying Lemma 4.6 and Lemma 4.7 repeatedly.

Proof of Lemma 4.5 (Sketch): Similar to Lemma 4.4, but in this case we also need Lemma 4.8 .

Let us now extend the relation $\rightarrow$ to proof nets as follows: $[P \triangleright \Gamma] \rightarrow[Q \triangleright \Delta]$ if an only if there are proof graphs $P^{\prime} \triangleright \Gamma$ and $Q^{\prime} \triangleright \Delta$ such that

$$
P \triangleright \Gamma \sim P^{\prime} \triangleright \Gamma \rightarrow Q^{\prime} \triangleright \Delta \sim Q \triangleright \Delta
$$


4.9 Lemma. There is no infinite sequence

$$
[P \triangleright \Gamma] \rightarrow\left[P^{\prime} \triangleright \Gamma^{\prime}\right] \rightarrow\left[P^{\prime \prime} \triangleright \Gamma^{\prime \prime}\right] \rightarrow \cdots
$$

Proof: In each reduction step the size of the sequent (i.e. the number of $\ngtr, \otimes$ and (D-nodes) is reduced.

4.10 Lemma. Let $P \triangleright \Gamma \sim P^{\prime} \triangleright \Gamma$, and let $P \triangleright \Gamma \rightarrow Q \triangleright \Delta$ and $P^{\prime} \triangleright \Gamma \rightarrow Q^{\prime} \triangleright \Delta$, i.e. in both reductions the same cut is reduced. Then we have $Q \triangleright \Delta \sim Q^{\prime} \triangleright \Delta$.

Proof: Easy case analysis.

4.11 Lemma. If $Q \triangleright \Delta \leftarrow P \triangleright \Gamma \rightarrow R \triangleright \Sigma$, then either $Q \triangleright \Delta=R \triangleright \Sigma$, or there is a proof graph $S \triangleright \Phi$ such that $Q \triangleright \Delta \rightarrow S \triangleright \Phi \leftarrow R \triangleright \Sigma$.

4.12 Lemma. If $[Q \triangleright \Delta] \leftarrow[P \triangleright \Gamma] \rightarrow[R \triangleright \Sigma]$, then either $[Q \triangleright \Delta]=$ $[R \triangleright \Sigma]$, or there is a proof net $[S \triangleright \Phi]$ such that $[Q \triangleright \Delta] \rightarrow[S \triangleright \Phi] \leftarrow[R \triangleright \Sigma]$.

Proof (Sketch): Let $\Phi_{1}$ denote the cut that is reduced in $\Gamma$ to obtain $\Delta$ and $\Phi_{2}$ the one that is reduced to obtain $\Sigma$. The basic idea is to apply Theorem 4.3 in order to make both cuts ready at the same time and then apply Lemma 4.11 and Lemma 4.10. There is essentially only one case in which it is not possible to make both cuts ready at the same time, namely, when they use the same axiom link. In other words, $P \triangleright \Gamma$ is of the following shape:

$$
\begin{gathered}
P^{\prime}\left\{\left(P^{\prime \prime}\left\{a_{h}^{\perp} \otimes a_{i}\right\} \& P^{\prime \prime \prime}\left\{a_{j}^{\perp} \otimes a_{k}\right\}\right) \& P^{\prime \prime \prime \prime}\left\{a_{l}^{\perp} \otimes a_{m}\right\}\right\} \\
\nabla \\
a_{i} \Phi_{1} a_{j}^{\perp}, a_{k} \Phi_{2} a_{l}^{\perp}, \Phi
\end{gathered}
$$

But whatever order of reduction is used, in both cases we get something of the shape $S^{\prime}\left\{a_{h}^{\perp} \otimes a_{m}\right\} \triangleright \Phi$.

4.13 Theorem. The cut elimination reduction $\rightarrow$ on proof nets is strongly normalizing. The normal forms are cut free proof nets.

Proof: Termination is provided by Lemma 4.9 and confluence follows from Lemma 4.12. That the normal form is cut free is ensured by Theorem 4.3 ,

\section{$5 \quad *_{\text {-Autonomy }}$}

For any formula $A$, we can provide an identity proof net $\operatorname{id}_{A}=\left[I_{A} \triangleright A^{\perp}, A\right]$, where $I_{A}$ is called the identity linking which is defined inductively on $A$ as follows:

$$
\begin{aligned}
& I_{a}=I_{a^{\perp}}=a \otimes a^{\perp} \\
& I_{\perp}=I_{1}=\perp \otimes 1 \\
& I_{A \ngtr B}=I_{A \otimes B}=I_{A} \diamond I_{B}
\end{aligned}
$$

Observe that we can have that $I_{A}=I_{A^{\perp}}$ because changing the order of the arguments of a $\otimes$ or $\ngtr$ in the linking of a proof graph does not change the proof net (see 2.4).

Furthermore, for any two proof nets $f=\left[P \triangleright A^{\perp}, B\right]$ and $g=\left[Q \triangleright B^{\perp}, C\right]$, we can define their composition $g \circ f$ to be the result of the cut elimination 
procedure to $\left[P \ngtr Q \triangleright A^{\perp}, B \oplus B^{\perp}, C\right]$. That this is well-defined and associative follows almost immediately from the strong normalization of cut elimination. We also have that $f \circ \operatorname{id}_{A}=f=\operatorname{id}_{B} \circ f$.

This gives rise to a category $\mathbf{P N}(\mathcal{A})$ whose objects are the MLL formulas built over $\mathcal{A} \cup \mathcal{A}^{\perp} \cup\{\perp, 1\}$, and whose arrows are the proof nets. More precisely, the arrows between two objects $A$ and $B$ are the (cut-free) proof nets $\left[P \triangleright A^{\perp}, B\right]$. The operation $\otimes$ on formulas can be extended to a bifunctor $\otimes: \operatorname{PN}(\mathcal{A}) \times$ $\mathbf{P N}(\mathcal{A}) \rightarrow \mathbf{P N}(\mathcal{A})$ by defining for two arrows $f=\left[P \triangleright A^{\perp}, B\right]$ and $g=[Q \triangleright$ $\left.C^{\perp}, D\right]$ the arrow $f \otimes g=\left[P \ngtr Q \triangleright A^{\perp} \ngtr C^{\perp}, B \otimes D\right]$. It can easily be seen that this bifunctor makes our category symmetric monoidal (with unit 1): The basic natural isomorphisms demanded by the definition (associativity, right unit, left unit, symmetry) are

$$
\begin{aligned}
\alpha_{A, B, C} & =\left[I_{A} 8 I_{B} \ngtr I_{C} \triangleright A^{\perp} 8\left(B^{\perp} \ngtr C^{\perp}\right),(A \otimes B) \otimes C\right] \\
\rho_{A} & =\left[\perp \otimes I_{A} \triangleright A^{\perp} 8 \perp, A\right] \\
\lambda_{A} & =\left[\perp \otimes I_{A} \triangleright \perp \ngtr A^{\perp}, A\right] \\
\sigma_{A, B} & =\left[I_{A} 8 I_{B} \triangleright A^{\perp} 8 B^{\perp}, B \otimes A\right]
\end{aligned}
$$

It is easy to check these are indeed proof nets, that $\alpha, \rho, \lambda$, and $\sigma$ are natural isomorphisms for all formulas $A, B$, and $C$, and that the corresponding diagrams (see BW99) commute.

Furthermore, we can exhibit the (contravariant) duality functor $(-)^{\perp}$ whose object function has already been defined. For an arrow $f=\left[P \triangleright A^{\perp}, B\right]: A \rightarrow B$ let $f^{\perp}=\left[P \triangleright B, A^{\perp}\right]: B^{\perp} \rightarrow A^{\perp}$. This determines a symmetric *-autonomous category structure Bar79, BW99. In particular, we define the bifunctor $->-$ as $A \ngtr B=\left(A^{\perp} \otimes B^{\perp}\right)^{\perp}$ and its unit object as $\perp=1^{\perp}$. The last thing to check is that we have the natural bijection

$$
\begin{gathered}
\operatorname{Hom}(A \otimes B, C) \cong \operatorname{Hom}\left(A, B^{\perp} \ngtr C\right) \\
{\left[P \triangleright A^{\perp} \ngtr B^{\perp}, C\right] \mapsto\left[P \triangleright A^{\perp}, B^{\perp} \ngtr C\right] .}
\end{gathered}
$$

\section{The Free *-Autonomous Category}

In this section we will show that the category of proof nets is the free symmetric *-autonomous category. Let $\mathcal{A}$ be a set and let $\eta_{\mathcal{A}}: \mathcal{A} \rightarrow \operatorname{Obj}(\mathbf{P N}(\mathcal{A}))$ be the function that maps every element of $\mathcal{A}$ to itself seen as atomic formula. To say that $\mathbf{P N}(\mathcal{A})$ is the free ${ }^{*}$-autonomous category generated by $\mathcal{A}$ amounts to saying that

6.1 Theorem. For any ${ }^{*}$-autonomous category $3^{3}\left(\mathcal{C}, \otimes, 1_{\mathcal{C}},(-)^{\perp}\right)$ and any map $G^{\circ}: \mathcal{A} \rightarrow \operatorname{Obj}(\mathcal{C})$, there is a unique functor $G: \operatorname{PN}(\mathcal{A}) \rightarrow \mathcal{C}$, preserving the ${ }^{*}$-autonomous structure, such that $G^{\circ}=\operatorname{Obj}(G) \circ \eta_{\mathcal{A}}$, where $\operatorname{Obj}(G)$ is the restriction of $G$ on objects.

The remainder of this section is devoted to a sketch the proof of this theorem. For this we will introduce the following notation.

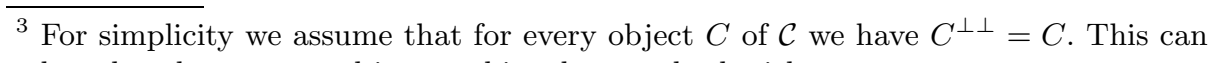
be relaxed to a natural isomorphism by standard trickery. 
Let $I$ be an index set. A bracketing of $I$ is given by a total order $I=$ $\left\{i_{1}, \ldots, i_{k}\right\}$ and a binary tree structure whose set of leaves is $I$, such that the order is respected. We will denote bracketings of $I$ also by $I$. The whole point of this is, given an $I$-indexed family $\left(C_{i}\right)_{i \in I}$ of objects of $\mathcal{C}$, that we can write $\bigotimes_{I}\left\{C_{i_{1}}, \ldots, C_{i_{k}}\right\}$ to denote the object of $\mathcal{C}$ that is obtained by applying the functor $-\otimes-$ according to the bracketing $I$. By a standard theorem of symmetric monoidal categories, any two objects obtained from different bracketings of the same set have a unique "coherence" isomorphism between them. Notice that this will involve the symmetry only if the order differs on the bracketings. Similarly, $\mho_{I}\left\{C_{i_{1}}, \ldots, C_{i_{k}}\right\}$ is defined. For empty $I$, let $\bigotimes_{\emptyset} \emptyset=1_{\mathcal{C}}$ and $\mho_{\emptyset} \emptyset=\perp_{\mathcal{C}}=1_{\mathcal{C}}^{\perp}$. The purpose of this notation is to state the following property of $*_{\text {-autonomous }}$ categories.

6.2 Proposition. Let $\mathcal{C}$ be $a^{*}$-autonomous category, and let $C_{1}, \ldots, C_{n}$ be objects of $\mathcal{C}$. Let $I, J \subseteq\{1, \ldots, n\}$, and let $\complement I=\{1, \ldots, n\} \backslash I$ and $\complement J=$ $\{1, \ldots, n\} \backslash J$ be their complements. Then for all bracketings of $I, J, \complement I, \complement J$, we have a natural bijection between $\operatorname{Hom}_{\mathcal{C}}\left(\otimes_{I}\left\{C_{i}^{\perp} \mid i \in I\right\}, \bigotimes_{\mathrm{C}_{I}}\left\{C_{i} \mid i \in \complement I\right\}\right)$ and $\operatorname{Hom}_{\mathcal{C}}\left(\bigotimes_{J}\left\{C_{i}^{\perp} \mid i \in J\right\}, \mathcal{\bigotimes}_{\mathrm{C} J}\left\{C_{i} \mid i \in \complement J\right\}\right)$.

Proof: The proof is done by repeatedly applying the associativity and commutativity of the two functors $-\otimes-$ and $-8-$, the natural isomorphisms for the units, and the natural bijection $\operatorname{Hom}_{\mathcal{C}}\left(A \otimes B^{\perp}, C\right) \cong \operatorname{Hom}_{\mathcal{C}}(A, B \ngtr C)$, which is imposed by the ${ }^{*}$-autonomous structure.

Let now the ${ }^{*}$-autonomous category $\mathcal{C}$ and the embedding $G^{\circ}: \mathcal{A} \rightarrow \operatorname{Obj}(\mathcal{C})$ be given. We will exhibit the functor $G: \operatorname{PN}(\mathcal{A}) \rightarrow \mathcal{C}$ which has the desired properties. On the objects, this functor is uniquely determined as follows:

$$
\begin{aligned}
& G(a)=G^{\circ}(a) \quad G(\perp)=\perp_{\mathcal{C}} \quad G(A \ngtr B)=G(A) \ngtr G(B) \\
& G\left(a^{\perp}\right)=G^{\circ}(a)^{\perp} \quad G(1)=1_{\mathcal{C}} \quad G(A \otimes B)=G(A) \otimes G(B)
\end{aligned}
$$

There is no other choice since the objects $1_{\mathcal{C}}$ and $\perp_{\mathcal{C}}$ in $\mathcal{C}$, as well as the functors $(-)^{\perp},-\otimes-$, and $-8-$ are uniquely determined by the ${ }^{*}$-autonomous structure on $\mathcal{C}$.

For defining $G$ on the morphisms, the situation is not as simple. We will first ignore the fact that the units are units and interpret a proof graph (with cuts) $P \triangleright \Gamma$ as an ordinary unit-free proof net with conclusions $A_{0}, \ldots, A_{n}, B_{1}$ (1) $B_{1}^{\perp}, \ldots, B_{m} \oplus B_{m}^{\perp}$, where $A_{0}=P^{\star}$ (see Observation 3.2 ) $, A_{1}, \ldots, A_{n}$ are the formulas in $\Gamma$ that are not cuts, and $B_{1} \oplus B_{1}^{\perp}, \ldots, B_{m} \oplus B_{m}^{\perp}$ are the cuts in $\Gamma$. To each such object we will uniquely assign a family of morphisms

$$
\bigotimes_{I}\left\{G\left(A_{i}\right)^{\perp} \mid i \in I\right\} \rightarrow \bigotimes_{\mathrm{C}_{I}}\left\{G\left(A_{i}\right) \mid i \in \complement I\right\}
$$

indexed by the bracketings on the subsets $I \subseteq\{0, \ldots, n\}$ and their complements. Proposition 6.2 ensures that every member of such a family of morphisms determines the others uniquely. The construction is done by induction on the size of the proof graph, using Lemma 3.3. (In fact, it is quite similar to the sequentialization.)

Observe that in particular this construction gives us for each proof graph $P \triangleright A^{\perp}, B$ a unique arrow $\psi_{P \triangleright A^{\perp}, B}: G\left(P^{\star}\right)^{\perp} \rightarrow G\left(A^{\perp}\right) \diamond G(B)$. Further- 
more, observe that for every linking $P$, the object $G\left(P^{\star}\right)^{\perp}$ in $\mathcal{C}$ is isomorphic to $\otimes_{a \otimes a^{\perp}}\left\{G(a) \& G(a)^{\perp}\right\}$, where $a \otimes a^{\perp}$ ranges over the axiom links in $P$. This means that the ${ }^{*}$-autonomous structure on $\mathcal{C}$ uniquely determines a morphism $\phi_{P}: 1_{\mathcal{C}} \rightarrow G\left(P^{\star}\right)^{\perp}$. This can be composed with $\psi_{P \triangleright A^{\perp}, B}$ to get $\xi_{\left[P \triangleright A^{\perp}, B\right]}: 1_{\mathcal{C}} \rightarrow G\left(A^{\perp}\right) \ngtr G(B)$. That this is well-defined, is ensured by the following lemma (in which we no longer ignore the fact that the units are units).

6.3 Lemma. If $Q \triangleright A^{\perp}, B \sim P \triangleright A^{\perp}, B$, then $\xi_{\left[P \triangleright A^{\perp}, B\right]}=\xi_{\left[Q \triangleright A^{\perp}, B\right]}$.

Consequently, to each proof net $f=\left[P \triangleright A^{\perp}, B\right]$, we can uniquely assign the arrow $G(f): G(A) \rightarrow G(B)$ that is determined by $\xi_{\left[P \triangleright A^{\perp}, B\right]}$ via Proposition 6.2 .

It remains to show that $G: \mathbf{P N}(\mathcal{A}) \rightarrow \mathcal{C}$ is indeed a functor (i.e. identities and composition are preserved). That for each formula $A$, the proof $\left[I_{A} \triangleright A^{\perp}, A\right]$ is mapped to identity id : $G(A) \rightarrow G(A)$ is an easy induction on the structure of $A$ and left to the reader. The preservation of composition is ensured by the following lemma.

6.4 Lemma. Let $T \triangleright \Gamma \rightarrow S \triangleright \Delta$, i.e. the proof graph $S \triangleright \Delta$ is obtained from $T \triangleright \Gamma$ by applying a single cut reduction step. Then $\xi_{[T \triangleright \Gamma]}$ and $\xi_{[S \triangleright \Delta]}$ denote the same morphism $1_{\mathcal{C}} \rightarrow \bigotimes\left\{G\left(A_{1}\right), \ldots, G\left(A_{n}\right)\right\}$, where $A_{1}, \ldots, A_{n}$ are the formulas in $\Gamma$ (resp. $\Delta)$ that are not cuts.

It might be worth mentioning, that Theorem 6.1 provides a decision procedure for the equality of morphisms in the free symmetric *-autonomous category, which is in our opinion simpler than the ones provided in BCST96] and [KO99.

\section{Conclusion}

We think we made a convincing case for the the cleanest approach yet to proof nets with the multiplicative units. There is always the possibility that another "ideology" than category theory will arise and will tell us to identify sequent proofs in a different way, perhaps collapsing fewer proofs, and help us construct more rigid proof objects. But we doubt very much that such a thing exists, given that the permutation rules that category theory imposes on the sequent calculus are so natural and so hard to weaken.

There are some issues that are left open and that we want to explore in the future:

- The relation with the new proof formalism called the calculus of structures GS01, BT01. We should mention that the idea behind our approach originates from the new viewpoints that are given by the calculus of structures.

- The addition of additives to our theory. This should not be very hard, given the work done in HvG03. The true challenge is to include also the additive units.

- The development of a theory of proof nets for classical logic. The problem is finding the right extension of the axioms of a *-autonomous category, such that on the one hand classical proofs are identified in a natural way, and on the other hand there is no collapse into a boolean algebra. 
- The search for meaningful invariants. It is very probable that the equivalence classes of graphs we define have a geometric meaning, and can be related to more abstract invariants like those given by homological algebra. We are convinced that the work in in [Mét94] is only the tip of the iceberg.

\section{References}

[Bar79] Michael Barr. *-Autonomous Categories, volume 752 of Lecture Notes in Mathematics. Springer-Verlag, 1979.

[BC99] Vincent Balat and Roberto Di Cosmo. A linear logical view of linear type isomorphisms. In Computer Science Logic, CSL 1999, volume 1683 of LNCS, pages 250-265. Springer-Verlag, 1999.

[BCST96] Richard Blute, Robin Cockett, Robert Seely, and Todd Trimble. Natural deduction and coherence for weakly distributive categories. Journal of Pure and Applied Algebra, 113:229-296, 1996.

[Blu93] Richard Blute. Linear logic, coherence and dinaturality. Theoretical Computer Science, 115:3-41, 1993.

[BT01] Kai Brünnler and Alwen Fernanto Tiu. A local system for classical logic. In R. Nieuwenhuis and A. Voronkov, editors, LPAR 2001, volume 2250 of Lecture Notes in Artificial Intelligence, pages 347-361. Springer-Verlag, 2001.

[BW99] Michael Barr and Charles Wells. Category Theory for Computing Science. Les Publications CRM, Montréal, third edition, 1999.

[DR89] Vincent Danos and Laurent Regnier. The structure of multiplicatives. Annals of Mathematical Logic, 28:181-203, 1989.

[Gir87] Jean-Yves Girard. Linear logic. Theoretical Computer Science, 50:1-102, 1987.

[Gir91] Jean-Yves Girard. A new constructive logic: Classical logic. Mathematical Structures in Computer Science, 1:255-296, 1991.

[GS01] Alessio Guglielmi and Lutz Straßburger. Non-commutativity and MELL in the calculus of structures. In Laurent Fribourg, editor, Computer Science Logic, CSL 2001, volume 2142 of LNCS, pages 54-68. Springer-Verlag, 2001.

[HvG03] Dominic Hughes and Rob van Glabbeek. Proof nets for unit-free multiplicative-additive linear logic. In 18'th IEEE Symposium on Logic in Computer Science (LICS 2003), 2003.

[KL71] Gregory Maxwell Kelly and Saunders Mac Lane. Coherence in closed categories. Journal of Pure and Applied Algebra, 1:97-140, 1971.

[KO99] Thong-Wei Koh and Chih-Hao Luke Ong. Internal languages for autonomous and *-autonomous categories. In Martin Hofmann, Giuseppe Rosolini, and Dusko Pavlovic, editors, Proceedings of the 8th Conference on Category Theory and Computer Science, Edinburgh, September 1999, volume 29 of Electronic Notes in Theoretical Computer Science. Elsevier, 1999.

[Laf95] Yves Lafont. From proof nets to interaction nets. In J.-Y. Girard, Y. Lafont, and L. Regnier, editors, Advances in Linear Logic, volume 222 of London Mathematical Society Lecture Notes, pages 225-247. Cambridge University Press, 1995.

[Mét94] François Métayer. Homology of proof nets. Archive of Mathematical Logic, 33:169-188, 1994.

[Thi03] Rüdiger Thiele. Hilbert's twenty-fourth problem. American Mathematical Monthly, 110:1-24, 2003. 